\title{
Single-Pill Combination of Perindopril/Indapamide/ Amlodipine in Patients with Uncontrolled Hypertension: A Randomized Controlled Trial
}

\author{
Sergey V. Nedogoda $\cdot$ Vesna J. Stojanov
}

Received: October 13, 2016 / Published online: February 8, 2017

(C) The Author(s) 2017. This article is published with open access at Springerlink.com

\begin{abstract}
Introduction: Patients with hypertension often require a combination of three antihypertensive agents to achieve blood pressure control, but very few single-pill triple combinations are available. The aim of this study was to determine whether a single-pill triple combination of perindopril, indapamide, and amlodipine was as effective as a dual-pill combination of perindopril/indapamide plus separate amlodipine at reducing blood pressure in patients with uncontrolled, essential hypertension.

Methods: This international, multicenter, open-label, randomized controlled trial was conducted in men or women aged $\geq 18$ years old with confirmed essential hypertension
\end{abstract}

Enhanced content To view enhanced content for this article go to http://www.medengine.com/Redeem/ A487F0607C763559.

Electronic supplementary material The online version of this article (doi:10.1007/s40119-017-0085-7) contains supplementary material, which is available to authorized users.

S. V. Nedogoda $(\varangle)$

Department Volgograd Medical State University, Volgograd, Russia

e-mail: nedogodasv@rambler.ru

\section{J. Stojanov}

Center for Hypertension, Clinical Center of Serbia, Medical School University of Belgrade, Belgrade, Serbia
(SBP $\geq 140$ and $<160 \mathrm{mmHg}$ and $\mathrm{DBP} \geq 90$ and $<100 \mathrm{mmHg}$ ), uncontrolled on maximal dose antihypertensive monotherapy or with a single dose of dual therapy. Patients were randomly assigned to: single-pill triple combination of perindopril $5 \mathrm{mg} /$ indapamide $1.25 \mathrm{mg} /$ amlodipine $5 \mathrm{mg}$ (Per/Ind/Aml) or dual-pill combination perindopril $5 \mathrm{mg}$ /indapamide $1.25 \mathrm{mg}+$ amlodipine $5 \mathrm{mg}$ (Per/Ind + Aml) once daily for 12 weeks. The primary endpoint was change in office supine SBP and DBP from baseline to week 12 . The proportion of responders defined as those with normalized BP (SBP $<140 \mathrm{mmHg}$ and DBP $<90 \mathrm{mmHg}$ ), and/or decrease of $S B P \geq 20 \mathrm{mmHg}$, and/or decrease of DBP $\geq 10 \mathrm{mmHg}$ at week 12 (W12) compared with baseline was also assessed. Secondary efficacy endpoints included change in office supine SBP and DBP, response, and BP control at weeks 4 and 8 . The tolerability of the treatments was also assessed.

Results: A total of 148 patients were randomized: 75 to Per/Ind/Aml and 73 to Per/ Ind + Aml. Mean supine SBP and DBP were $149.1 \pm 4.7$ and $94.1 \pm 3.1 \mathrm{mmHg}$, respectively, with no relevant between-group difference. At week 12, both triple-therapy regimens were associated with clinically significant reductions in SBP compared with baseline $(-21.5 \pm 11.7$ and $-20.0 \pm 12.9 \mathrm{mmHg}$, respectively). Reductions in office supine DBP were also clinically significant $\quad(-15.3 \pm 7.8$ and $-14.8 \pm 9.0$ $\mathrm{mmHg}$, respectively). The proportion of 
treatment responders was high in both groups: 89.2 and $87.1 \%$, respectively. The reduction in office supine SBP/DBP was already evident at week 4 and maintained for the duration of the study in both groups. The majority of patients were treatment responders at week 4 (89.2 and $82.9 \%$, respectively) and had achieved BP control ( 87.8 vs. $78.6 \%$, respectively), which was maintained until week 12 in both treatment groups. Both treatments were well tolerated with no between-group differences.

Conclusions: In adult patients with uncontrolled essential hypertension on treatment, single-pill triple-combination therapy with Per/Ind/Aml is as effective as the same dose dual-pill combination of Per/Ind + Aml. Both treatments were associated with clinically significant BP reductions compared with baseline and were well tolerated.Clinical trials number: http://www.controlled-trials.com ISRCTN: 16442558.Funding: Les Laboratoires Servier.

Keywords: Amlodipine; Antihypertensive; Indapamide; Perindopril; Single-pill combination; Uncontrolled essential hypertension

\section{INTRODUCTION}

Hypertension is a major modifiable risk factor for cardiovascular disease and stroke and there is global agreement among current hypertension management guidelines that the majority of patients with uncomplicated hypertension should be treated to a blood pressure (BP) goal of systolic blood pressure (SBP) $<140 \mathrm{mmHg}$ and diastolic blood pressure (DBP) $<90 \mathrm{mmHg}$ [1-6].

Monotherapy can effectively reduce BP in only a limited number of patients and the majority will therefore require treatment with two or more agents to reach target levels [7]. In a meta-analysis of SBP reductions in 42 trials $(N=10,698)$, the combination of any two medications from different BP-lowering drug classes was approximately five times more effective than doubling the dose of a single drug [8].

The use of triple-combination therapy, targeting three different mechanisms of arterial hypertension pathogenesis, is becoming increasingly used to provide optimal BP control [8-13] with fewer dose-related adverse effects. In addition, each agent in the combination can lessen adverse effects of other components [14]. This may counteract the tendency of some physicians to prescribe lower than guideline-recommended doses of anti-hypertensive drugs because of concerns over tolerability, and the inevitable suboptimal dosing and inadequate dose titration required to achieve target BP goals [15, 16].

When triple-combination therapy is required, the European Society of Cardiology/ European Society of Hypertension guidelines [1] and other guidelines [3,17-20] recommend the use of an angiotensin-converting enzyme (ACE) system inhibitor or angiotensin II receptor blocker (ARB) with a calcium channel blocker (CCB) and a diuretic as rational and effective. One such combination is perindopril, indapamide, and amlodipine. In addition to additive effects on BP control, each component of this combination has demonstrated protection of target organs at risk from hypertension including the heart, renal system, brain, and vasculature, in a wide range of patients with hypertension. The three components are also complementary in terms of tolerability: perindopril reduces the peripheral edema that is a dose-limiting side effect of CCBs [14]; amlodipine reduces ACE inhibitor-related cough by decreasing prostaglandin synthesis that is induced by bradykinin [21]; and indapamide is a metabolically neutral diuretic [22]. Each individual component is associated with a large evidence base of use in arterial hypertension, and single-pill combinations have also been extensively evaluated: perindopril/indapamide has shown efficacy in patients with recurrent stroke [23], diabetes mellitus [24], and in octogenarians with hypertension [25]; perindopril/amlodipine combination has been studied in detail in patients with hypertension at high cardiovascular risk [26].

Given the extensive experience with these agents as mono and dual therapy it was hypothesized that combining the three agents into a single-pill would maintain the established efficacy and safety of these agents while reducing dosing complexity. Knowing that 
some physicians may be reluctant to prescribe fixed-dose triple-therapy combinations due to concerns over tolerability, the aim of the present study was to evaluate the clinical efficacy and safety of a triple combination of perindopril $5 \mathrm{mg}$ /indapamide $1.25 \mathrm{mg}$ /amlodipine $5 \mathrm{mg}$ in a single-pill versus dual-pill combination perindopril $5 \mathrm{mg}$ /indapamide $1.25 \mathrm{mg}$ plus amlodipine $5 \mathrm{mg}$ over 12 weeks, in treated patients with uncontrolled hypertension.

\section{METHODS}

\section{Study Design}

The study was an international, multicenter, open-label, randomized controlled trial conducted at 13 centers in Russia and four centers in Serbia. The study protocol was approved by independent Ethics Committees, in accordance with the local regulations in each of the countries and complied with the Declaration of Helsinki, current Good Clinical Practice guidelines (including source documents archiving), and local laws and regulations (ISRCTN registration number: 16442558). All participants provided informed consent before enrolment. Patients were randomized 1:1 to treatment by a computer-generated allocation schedule using a permuted block method (with a block size of four) by the clinical biostatistics department of the study sponsor. Randomization was stratified by study center.

\section{Participants}

Eligible patients were men or women of any ethnic origin, aged $\geq 18$ years old, treated with antihypertensive monotherapy at maximal dose or with a single dose of dual therapy other than study treatment (perindopril or indapamide or amlodipine), and with confirmed essential, uncontrolled, hypertension on treatment $(\mathrm{SBP} \geq 140$ and $<160 \mathrm{mmHg}$ and $\mathrm{DBP} \geq 90$ and $<100 \mathrm{mmHg}$ at two separate visits: selection and inclusion). During the run-in period (1 week) patients continued their current antihypertensive therapy. Uncontrolled hypertension was confirmed at the inclusion visit. Patients were excluded if they had any contraindications to perindopril, indapamide or amlodipine, or any of the following conditions: complicated hypertension (known stage III or IV hypertensive retinopathy); macroalbuminuria; diabetes; moderate or severe renal failure (creatinine clearance 30-59 and $<30 \mathrm{ml} / \mathrm{min}$, respectively); known complicated liver disease; recent disease (previous 6 months) such as cerebrovascular disease (ischemic stroke, cerebral hemorrhage, transient ischemic attack); recent ventricular rhythm disorders (except isolated extrasystoles); and known or suspected symptomatic orthostatic hypotension (defined as a reduction in $\mathrm{SBP} \geq 20 \mathrm{mmHg}$ and/or in $\mathrm{DBP} \geq 10 \mathrm{mmHg}$ from supine to standing-up, maintained for the first 3 min of standing-up).

\section{Intervention}

At the inclusion visit, patients were randomly assigned to one of the following treatment groups: a single-pill triple combination of perindopril $5 \mathrm{mg} /$ indapamide $1.25 \mathrm{mg} / \mathrm{am}$ lodipine $5 \mathrm{mg}$ ( 1 tablet/day orally in the morning before breakfast), or a dual-pill combination of perindopril $5 \mathrm{mg} /$ indapamide $1.25 \mathrm{mg}+$ amlodipine $5 \mathrm{mg}$ ( 1 tablet each/day orally in the morning before breakfast) (Fig. 1). Treatment allocation was balanced, non-centralized, non-adaptive and stratified by center. It was performed according to the allocation list sent to each site (blocks of size 4). The treatment numbers were assigned in order, sequentially, according to the list. The treatment period was 12 weeks.

\section{Study Assessments}

All office BP measurements were witnessed and performed at each visit (weeks $0,4,8,12$ ), on the same arm, using a validated automatic OMRON blood pressure measurement device (Model 705CP-II, Omron Healthcare Inc., Vernon Hills, IL, USA) according to ESH guidelines [1]: BP was recorded three times at 1-2-min intervals in the supine position after $10 \mathrm{~min}$ of rest. The mean of the last two consecutive measurements was used as the BP level at the 

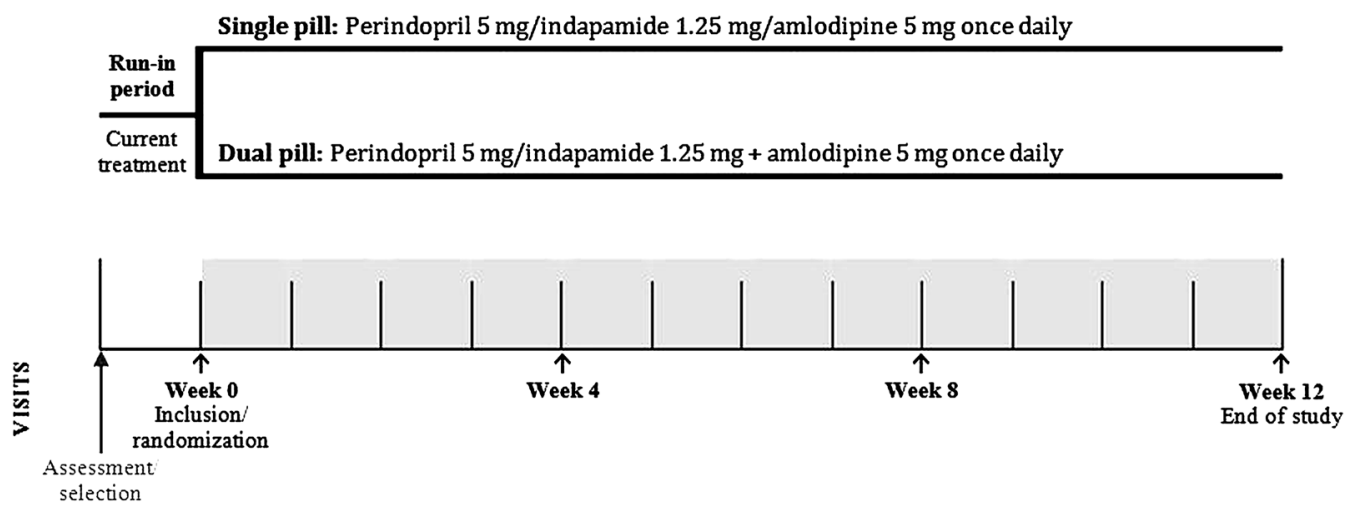

Fig. 1 Study design

study visit. Office SBP and DBP values were measured at baseline prior to the first drug intake and at all post-baseline visits at trough (i.e., $24 \pm 3 \mathrm{~h}$ after the last intake). The response criteria were the proportion of patients with normalized BP (SBP $<140 \mathrm{mmHg}$ and $\mathrm{DBP}<90$ $\mathrm{mmHg}$ ), and/or decrease of $\mathrm{SBP} \geq 20 \mathrm{mmHg}$, and/or decrease of $\mathrm{DBP} \geq 10 \mathrm{mmHg}$ at week 12 (W12) compared with baseline. In order to identify orthostatic hypotension, two additional measurements of SBP and DBP in a standing position were performed 1 and $3 \mathrm{~min}$ after rising from a supine position.

Safety assessments were performed at inclusion and W12 and included complete laboratory examinations by local laboratories: blood biochemistry; hematology and urine check for proteinuria. Dyslipidemia was qualified by the investigator based on the patient's medical history or according to treatment or laboratory values and coded using the MedDRA dictionary (version 17.0). Total cholesterol was evaluated during the study (at baseline and at study end) and any significant abnormal value was reported as an adverse event. At W4 only creatinine, creatinine clearance, uric acid and potassium were assessed. Orthostatic hypotension and heart rate were evaluated at every visit.

\section{Statistical Methods}

Descriptive statistics were provided for study outcome and safety analyses. The full analysis set (FAS) was used for evaluation of the primary endpoint. Based on the intention-to-treat principle, the FAS corresponds to randomized patients having received at least one dose of study treatment and having a value at baseline and at least one post-baseline value for office supine SBP and DBP over the W0-W12 period. The per protocol set (PPS) corresponds to patients from the FAS without relevant protocol violation(s) that could have affected the evaluation of supine SBP and DBP criteria.

For all primary and secondary endpoints, the treatment effect was estimated, as well as its accuracy: estimate of the between-group difference, standard error of the estimate, and two-sided $95 \%$ confidence interval (CI) of the estimate.

The primary efficacy outcome was change from baseline to last post-baseline value in office supine SBP and DBP over the WO-W12 period. The response to antihypertensive treatment expressed as the rate of responders at last post-baseline visit over W0-W12 period was also assessed.

A number of secondary efficacy endpoints were also evaluated including: change from baseline to post-baseline value in office supine SBP and DBP over the WO-W4 and WO-W8 periods; response to antihypertensive treatment expressed as the rate of responders at each post-baseline visit over the W0-W4 and W0-W8 periods; and BP control expressed as the proportion of patients with BP control at post-baseline visit over W0-W12 period and at each post-baseline visit $\mathrm{W} 4$ and $\mathrm{W} 8$. 


\section{Compliance with Ethics Guidelines}

All procedures followed were in accordance with the ethical standards of the responsible committee on human experimentation (institutional and national) and with the Helsinki Declaration of 1964, as revised in 2013. Informed consent was obtained from all patients for being included in the study.

\section{RESULTS}

A total of 148 patients were randomized: 75 to the perindopril $5 \mathrm{mg} /$ indapamide $1.25 \mathrm{mg} / \mathrm{am}$ lodipine $5 \mathrm{mg}$ group (Per/Ind/Aml) and 73 to the perindopril $5 \mathrm{mg} /$ indapamide $1.25 \mathrm{mg}+$ amlodipine $5 \mathrm{mg}$ group (Per/Ind $+\mathrm{Aml}$ ). The number of patients completing the study was 134: 70 (Per/Ind/Aml) and 64 (Per/Ind + Aml). Fourteen patients were withdrawn from the study (five in the Per/Ind/Aml group and nine in Per/Ind $+\mathrm{Aml})$. The main reason for withdrawal was protocol deviation for 10 patients (three in Per/Ind/Aml, seven in Per/Ind + Aml). The number of patients withdrawn due to adverse events was low (1 patient in each treatment group). Protocol deviations were mainly related to timing of blood pressure measures with no relevant between-group differences. The trial profile is illustrated in Fig. 2.

\section{Baseline Characteristics}

Baseline characteristics are summarized in Table 1 and no major differences were observed between the two groups. Mean age was 56 years and a large proportion was at least 60 years old (44\%). Just over half of the patients were women (53\%). All patients presented with essential hypertension with an average duration of 99 months (range 2-471 months). A family history of hypertension was reported in $70 \%$ of patients. Other relevant medical history included a higher frequency of dyslipidemia in the Per/Ind/Aml group than in the Per/Ind + Aml group: 53.3 versus $43.8 \%$. Frequent cardiovascular risk factors included smoking (18\%) and alcohol consumption (24\%).
At inclusion, all patients were receiving at least one antihypertensive treatment $(27.8 \%$ monotherapy and $72.2 \%$ dual therapy). The most common were agents acting on the renin-angiotensin system (96\%) and diuretics (65\%). Other concomitant treatments were mainly lipid modifying agents (29\%) and antithrombotic agents (22\%).

No relevant between-group differences were observed in vital signs. Mean supine SBP and DBP at inclusion were 149 and $94 \mathrm{mmHg}$, respectively. Mean heart rate was $77 \mathrm{bpm}$.

\section{Adherence}

The mean treatment duration was $82.2 \pm 13.8$ days for the period W0-W12 in the FAS. Mean treatment adherence was very good at $97.6 \pm 6.4 \%$, and all patients but one had an overall adherence between 80 and 120\%. No relevant between-group differences were observed in adherence.

\section{Primary Endpoints}

A decrease in office supine SBP was observed in both treatment groups for last post-baseline value compared with baseline, even though patients were already receiving antihypertensive therapy before entry into the study (monotherapy at maximal dose or a single dose of dual therapy other than study treatment). The changes were similar in both treatment groups: $-21.5 \pm 11.7 \mathrm{mmHg}$ in the Per/Ind/Aml group versus $-20.0 \pm 12.9 \mathrm{mmHg}$ in the Per/ Ind + Aml group; mean (SE) between-group difference in office supine SBP -1.4 (1.8) $\mathrm{mmHg}, 95 \%$ CI $[-5.1 ; 2.2]$ (Table 2) (Fig. 3).

Results for office supine DBP followed the same trend with a decrease over time and similar changes in both treatment groups: $-15.3 \pm 7.8 \mathrm{mmHg}$ in the Per/Ind/Aml group versus $-14.8 \pm 9.0 \mathrm{mmHg}$ in the Per/Ind $+\mathrm{Aml}$ group; mean (SE) between-group difference -0.5 (1.3) $\mathrm{mmHg}, 95 \%$ CI $[-3.1 ; 2.1]$ (Table 2) (Fig. 3). For both office supine SBP and DBP results for the per protocol set (PPS) were similar to the FAS. 


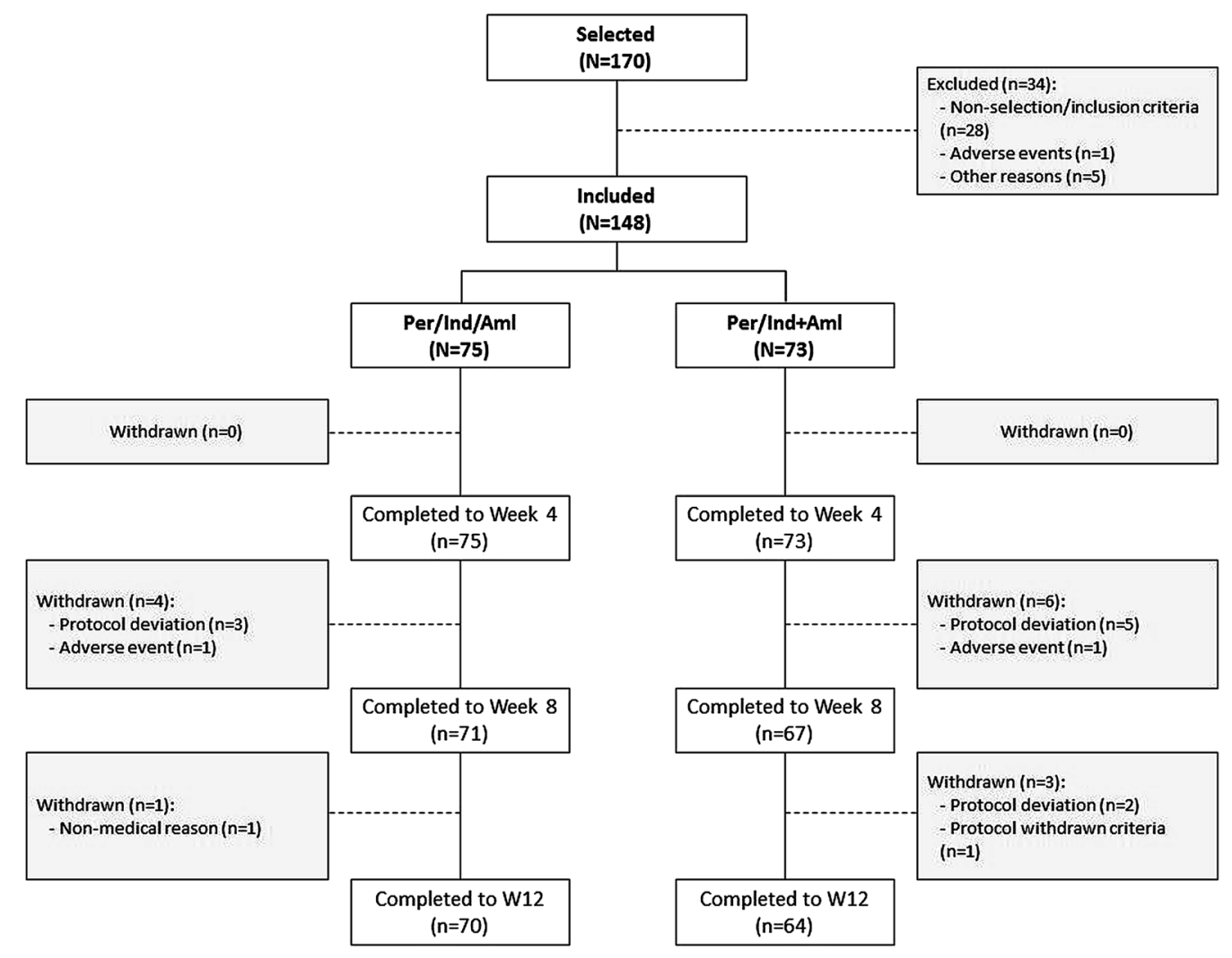

Fig. 2 Trial profile illustrating selected/included patients

A response to antihypertensive treatment at the last post-baseline value was observed for the majority of patients in both treatment groups, and the frequency of responders was similar: $89.2 \%$ in the Per/Ind/Aml group versus $87.1 \%$ in the Per/Ind + Aml group; mean (SE) between-group difference $2.1 \%(5.4)$, 95\% CI $[-8.5 ; 12.6]$ in the FAS. A similar trend was observed in the PPS.

Similar changes in SBP and DBP were also observed in the two treatment groups for the monotherapy and dual therapy subgroups (Supplementary Tables S1 and S2).

\section{Secondary Endpoints}

For the secondary endpoints at intermediate visits, results for the PPS were similar to the FAS and only the latter are presented. Most of the changes in SBP and DBP were observed by the first visit at week 4 and maintained over the course of the study, and were similar in both groups (Supplementary Figures S1 A and S1B). At W4, SBP decreased by $-19.8 \mathrm{mmHg}$ in the $\mathrm{Per} / \mathrm{Ind} / \mathrm{Aml}$ group versus $-19.0 \mathrm{mmHg}$ in the Per/Ind + Aml group; mean (SE) between-group difference -0.7 (1.6) $\mathrm{mmHg}, 95 \% \mathrm{CI}[-3.9 ; 2.5]$. For DBP, the corresponding values were -14.6 versus $-14.7 \mathrm{mmHg}$, respectively; mean (SE) between-group difference 0.04 (1.32) $\mathrm{mmHg}$, 95\% CI $[-2.6 ; 2.7]$. These decreases in BP were maintained throughout the study. Accordingly, most patients were responders from the W4 visit (89.2 versus $82.9 \%$, respectively; mean (SE) between-group difference $6.3 \%$ (5.8), 95\% CI $[-5.0 ; 17.7]$. At W8, the percentage of responders was $91.9 \%$ versus $92.9 \%$, respectively; mean (SE) between-group difference $-1.0 \%$ (4.4), 95\% CI [-9.6; 7.7].

Control of supine BP (defined as SBP $<140 \mathrm{mmHg}$ and DBP $<90 \mathrm{mmHg}$ ) was reached in most patients in both treatment groups for the post-baseline value at $\mathrm{W} 4, \mathrm{~W} 8$, and $\mathrm{W} 12$. In the majority of patients, control of supine BP 
Table 1 Baseline demographic characteristics in the randomized set

\begin{tabular}{|c|c|c|c|}
\hline Baseline characteristic & Per/Ind/Aml $(n=75)$ & Per $/$ Ind + Aml $(n=73)$ & Global $(n=148)$ \\
\hline Age (years) & $57.2 \pm 10.6$ & $55.6 \pm 10.9$ & $56.4 \pm 10.8$ \\
\hline Over 60 years (\%) & 46.7 & 41.1 & 43.9 \\
\hline Women (\%) & 53.3 & 52.1 & 52.7 \\
\hline BMI $\left(\mathrm{kg} / \mathrm{m}^{2}\right)$ & $27.8 \pm 3.3$ & $27.9 \pm 2.9$ & $27.8 \pm 3.1$ \\
\hline Duration of hypertension (months) & $103.5 \pm 89.7$ & $94.1 \pm 78.7$ & $98.9 \pm 84.3$ \\
\hline Family history of hypertension (\%) & 68.0 & 72.6 & 70.3 \\
\hline Dyslipidemia (\%) & 53.3 & 43.8 & 48.7 \\
\hline Smoker (\%) & 18.7 & 17.8 & 18.2 \\
\hline Alcohol consumption (\%) & 26.7 & 20.6 & 23.7 \\
\hline \multicolumn{4}{|c|}{ Previous treatment for hypertension (\%) } \\
\hline Monotherapy (\%) & 31.1 & 24.3 & 27.8 \\
\hline Bi-therapy (\%) & 68.9 & 75.7 & 72.2 \\
\hline \multicolumn{4}{|l|}{ Agents involved (\%) } \\
\hline ACEI or ARB & 93.3 & 98.6 & 95.9 \\
\hline Diuretic & 61.3 & 68.5 & 64.9 \\
\hline $\mathrm{CCB}$ & 14.7 & 9.6 & 12.2 \\
\hline \multicolumn{4}{|l|}{ Concomitant treatment } \\
\hline Lipid modifying agents & 32.0 & 26.0 & 29.1 \\
\hline Antithrombotic agents & 22.7 & 21.9 & 22.3 \\
\hline
\end{tabular}

was attained at W4 and maintained until W12 in both treatment groups. At W4, 87.8\% of patients in the Per/Ind/Aml group versus $78.6 \%$ in the Per/Ind + Aml group achieved control of supine BP; mean (SE) between-group difference 9.3\% (6.20), 95\% CI [-2.89; 21.43]. W8 values were 85.1 versus $88.6 \%$, respectively; mean (SE) between-group difference $-3.4 \%$ (5.62), 95\% CI [-14.45; 7.57]. W12 values were 81.1 versus $80.0 \%$, respectively; mean (SE) between-group difference $1.1 \%$ (6.60), 95\% CI [-11.86; 14.02].

\section{Safety}

Safety was evaluated in all patients who took at least one dose of the study drug (safety set).
Emergent adverse events (EAEs) were reported in $18.7 \%$ patients in the Per/Ind/Aml group and $21.9 \%$ patients in the Per/Ind + Aml group. The most frequent EAEs in the Per/Ind/Aml group were hypokalemia (2.7\%) and nasopharyngitis $(2.7 \%)$, and in the Per/Ind + Aml group the most frequent EAEs were hyperuricemia, and an increase in potassium, and blood urea $(2.7 \%$ each); none were serious. There were no reports of peripheral edema or cough.

EAEs leading to treatment withdrawal were reported in one patient in each treatment group: hypertension in the Per/Ind/Aml group and rash (considered treatment-related) in the Per/Ind + Aml group. Neither event was serious and both patients recovered. 
Table 2 Change in office supine blood pressure from baseline to week 12

\begin{tabular}{|c|c|c|}
\hline & Per/Ind/Aml $(n=74)$ & Per/Ind + Aml $(n=70)$ \\
\hline \multicolumn{3}{|l|}{$\mathrm{SBP}(\mathrm{mm} \mathrm{Hg})$} \\
\hline Baseline $($ mean $\pm \mathrm{SD})$ & $149.1 \pm 4.9(95 \%$ CI $[147.9 ; 150.1])$ & $149.0 \pm 4.7(95 \%$ CI $[147.9 ; 150.1])$ \\
\hline Week 12 & $127.6 \pm 11.1(95 \%$ CI $[125.0 ; 130.1])$ & $129.0 \pm 11.7(95 \%$ CI $[126.2 ; 131.8])$ \\
\hline Week 12-baseline & $-21.5 \pm 11.7(95 \%$ CI $[-24.3 ;-18.8])$ & $-20.0 \pm 12.9(95 \% \mathrm{CI}[-23.1 ;-17.0])$ \\
\hline Estimate $^{a} \pm S D$ & $-1.4 \pm 1.8(95 \%$ CI $[-5.1 ; 2.2])$ & \\
\hline \multicolumn{3}{|l|}{ DBP (mm Hg) } \\
\hline Baseline (mean $\pm \mathrm{SD})$ & $94.1 \pm 3.0(95 \%$ CI $[93.4 ; 94.8])$ & $94.1 \pm 3.1(95 \%$ CI [93.4; 94.9]) \\
\hline Week 12 & $78.8 \pm 8.0(95 \%$ CI $[76.9 ; 80.6])$ & $79.3 \pm 8.4(95 \%$ CI $[77.3 ; 81.3])$ \\
\hline Week 12-baseline & $-15.3 \pm 7.8(95 \%$ CI $[-17.1 ;-13.5])$ & $-14.8 \pm 9.0(95 \%$ CI $[-17.0 ;-12.7])$ \\
\hline Estimate $^{\mathrm{a}} \pm \mathrm{SD}$ & $-0.5 \pm 1.3(95 \%$ CI $[-3.1 ; 2.0])$ & \\
\hline
\end{tabular}

Comparison between groups during the study period (week 12-baseline) for the full analysis set $(n=144)$

Aml amlodipine, CI confidence interval, Per perindopril, Ind indapamide, SD standard deviation

a Estimate ( \pm standard deviation) of the difference in adjusted mean changes from baseline to last post-baseline value until week $12[\mathrm{Per} / \mathrm{Ind} / \mathrm{Aml}]-[\mathrm{Per} / \mathrm{Ind}+\mathrm{Aml}]$ using a general linear model with treatment, baseline, and center as covariates

In this study, patients were carefully followed for any signs of emergent hypotension and orthostatic hypotension. Only one patient reported an emergent hypotension in the Per/ Ind + Aml group. This EAE was not serious and was not considered treatment-related. None of the patients were affected by a symptomatic orthostatic hypotension.

Treatment-related EAEs were reported in only a few patients: (4.0\%) in the Per/Ind/Aml group and $(6.8 \%)$ in the Per/Ind + Aml group. Those that were reported were in accordance with those listed in the summary of product characteristics for perindopril, indapamide, and amlodipine.

Blood biochemistry results showed that the rate of patients affected by potentially clinically significant abnormal (PCSA) values was low in both treatments groups $(<5 \%)$ and mostly detected for creatinine clearance (low value, whatever the formula used) in (4.2\%) patients in the Per/Ind/Aml group and (4.4\%) patients in the Per/Ind + Aml group. However, all these patients already had a baseline creatinine clearance $<90 \mathrm{ml} / \mathrm{min}$ or $\mathrm{ml} / \mathrm{min} / 1.73 \mathrm{~m}^{2}$ and were aged $\geq 60$ years except for one patient aged less than 60 years in the Per/Ind + Aml group.

\section{DISCUSSION}

As expected, this study confirms the similar and clinically significant efficacy in reducing office supine BP of both regimens with reductions of 21.5 and $20.0 \mathrm{mmHg}$ (SBP) and 15.3 and 14.8 (DBP) for the Per/Ind/Aml and Per/Ind + Aml groups, respectively. These reductions were achieved in a population with treated hypertension remaining uncontrolled on maximal dose monotherapy or single-dose dual therapy (mean baseline 149.1/94.1 $\mathrm{mmHg}$ ), and both treatment groups used low doses of the active components (perindopril $5 \mathrm{mg}$, indapamide $1.25 \mathrm{mg}$, amlodipine $5 \mathrm{mg}$ ). The majority of patients were responders: $89.2 \%$ (Per/Ind/Aml) and $87.1 \%(\mathrm{Per} / \mathrm{Ind}+\mathrm{Aml})$. The results indicate that therapy with therapeutic doses of three component antihypertensive therapies: ACE inhibitor (perindopril), CCB (amlodipine) and diuretic (indapamide) allows BP targets to be achieved in eight out of ten patients previously uncontrolled on mono or dual therapy.

Analysis of the secondary endpoints revealed that achievement of BP control was rapid. The full effect on supine SBP and DBP was attained by $\mathrm{W} 4$ and then maintained until the end of the 


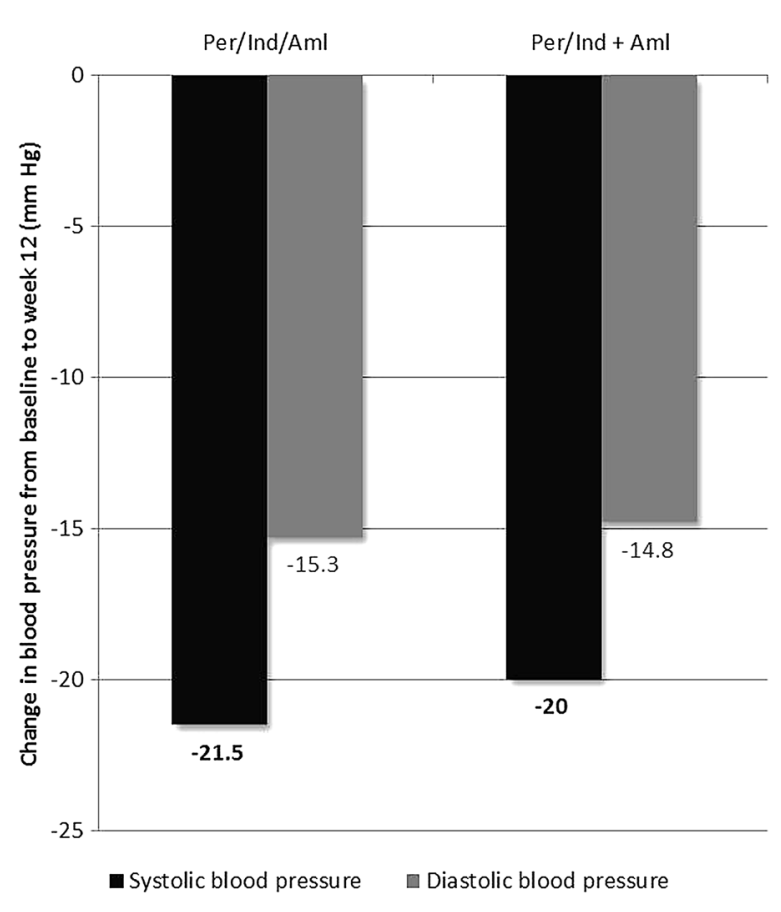

Fig. 3 Change in office supine blood pressure from baseline to week 12 with triple-drug antihypertensive therapy containing perindopril, indapamide, and amlodipine in patients with uncontrolled hypertension (SBP $\geq 140$ and $<160 \mathrm{mmHg}$ and $\mathrm{DBP} \geq 90$ and $<100 \mathrm{mmHg}$ at two separate visits: selection and inclusion). Comparison between single-pill (Per/Ind/Aml, $n=74$ ) and dual-pill combinations (Per/Ind $+\mathrm{Aml}, n=70)$. Aml amlodipine, Per perindopril, Ind indapamide

study. The percentage of responders was already very high at W4, with a rate of $89 \%$ in the Per/ Ind/Aml group compared with $83 \%$ in the Per/ Ind + Aml group, and more importantly the rate of hypertension control was 88 versus $79 \%$, respectively. Rapid attainment of target BP levels is an important goal of therapy and can lead to improved cardiovascular outcomes. Results from several major cardiovascular clinical trials suggest that the risk of vascular events is significantly influenced by the time it takes to achieve BP control [26-29].

Both triple-therapy regimens showed good tolerability and safety in the current study. The adverse events reported were in accordance with the known safety profile of the component drugs (perindopril, indapamide and amlodipine). Importantly there was no increased risk of hypotension with the use of triple therapy and no report of any peripheral edema or cough.

The study confirms previous observations from the PIANIST study [22] conducted in a high-risk population, in whom target BP levels were also achieved in more than $80 \%$ patients who had previously taken two antihypertensive drug combinations, albeit at higher doses of the three components than in the present study. The results suggest that the triple-therapy Per/ Ind/Aml combination is suitable for a wide spectrum of patients with hypertension at both low and high cardiovascular risk.

Neither the present study nor the PIANIST trial was designed to evaluate long-term outcomes. However, a post hoc analysis of data from ADVANCE (Action in Diabetes and Vascular Disease: Preterax and Diamicron Controlled Evaluation) hints at possible advantages of a triple combination. In the blood pressure arm of ADVANCE, 11, 140 patients with type 2 diabetes were randomly assigned to a fixed combination of perindopril/indapamide (4/ $1.25 \mathrm{mg}$ ) or placebo. Of these, 3427 patients were also receiving a CCB at baseline [30]. Active treatment reduced the relative risk of death by $28 \%(95 \%$ CI $[10 \% ; 43 \%])$ among patients with CCB at baseline compared with $5 \%(-12$ to $20 \%)$ among those without CCB $(P$ homogeneity $=0.02)$. The relative risk reduction for major cardiovascular events was $12 \%$ ( -8 to $28 \%$ ) versus $6 \%$ ( -10 to $19 \%$ ) for those with and without $\mathrm{CCB}$ at baseline although the difference was not statistically significant $(P$ homogeneity $=0.38)$. The benefit on total and cardiovascular death was seen independent of BP reduction because the group on CCBs had a $4.7 \mathrm{~mm} \mathrm{Hg}$ SBP reduction, whereas the non-CCB group had a $6.2 \mathrm{~mm} \mathrm{Hg}$ reduction. The treatment benefits were observed despite a higher risk profile among the patients receiving $\mathrm{CCB}$, including a longer duration of type 2 diabetes, more elevated SBP and DBP, higher rates of antihypertensive drug treatment, and higher rates of previous cardiovascular disease. There was no detectable increase in adverse effects in those receiving a CCB.

The greater BP reductions achieved with antihypertensive drug combinations means 
that lower drug doses are required to reach BP goals with subsequent minimized adverse effects. Furthermore, one component of an antihypertensive combination therapy can reduce the occurrence of adverse events caused by a second component with a different mechanism of action. For example, thiazide diuretics lower BP by reducing volume, which may activate the renin-angiotensin-aldosterone system (RAAS) as a compensatory mechanism and in turn raise BP; a RAAS blocker counteracts this effect and provides additive BP control. RAAS blockers may also reduce the risk of CCB-induced edema by beneficial effects on post-capillary dilation and hydrostatic pressure [31].

There are currently two other approved single-pill triple-therapy combinations available in Europe: olmesartan/amlodipine/hydrochlorothiazide [32] and valsartan/amlodipine/ hydrochlorothiazide [33]. However, these agents have no long-term outcome data and neither contains a diuretic that has proven efficacy in a clinical outcome trial, such as indapamide $[23,25,30]$ which is recommended by cardiology experts because of its strong morbidity and mortality data [34-38]. As the first single-pill triple-therapy combination incorporating an ACE inhibitor, Per/Ind/Aml fulfills a therapeutic need for many patients currently benefiting from an ACE-inhibitor based therapy who require an additional therapy with single pill. Clinically, ACE inhibitors and ARBs may appear similar. Both are used to treat cardiovascular risk factors and both reduce $\mathrm{BP}$, stroke and symptoms of heart failure. However, the existence of clinical differences, in particular with regards to cardiovascular risk reduction, have been demonstrated by a number of authors. Recently, several meta-analyses have compared the effects of ACE inhibitors and ARBs demonstrating that ACE inhibitors prevent coronary events with reductions in cardiovascular morbidity and mortality endpoints while ARBs are, at best, efficient for the prevention of stroke [39]. One of these, which was fundamentally robust in terms of data quality and numbers analyzed (73,100 patients), showed that ACE inhibitors, but not ARBs, were associated with mortality reduction [40]. This finding was confirmed in a further meta-analysis, performed in 108,212 patients without heart failure, but at high cardiovascular risk [41]. Parallel meta-analyses of ACE inhibitor and ARB trials vs. placebo or other active comparator, and meta-regression analyses that have adjusted for BP within the trials, have demonstrated that ACE inhibitors reduce the risk of myocardial infarction and mortality above and independently of BP lowering, whereas ARBs do not, reinforcing the need for an ACE inhibitor in a triple-therapy combination [42]. The meta-analysis of Ferrari et al. showed that perindopril-based trials accounted for a substantial part of the all-cause and cardiovascular mortality reduction observed with RAAS inhibitors in hypertension [39].

A further benefit of a single-pill triple combination is that it reduces the complexity of the treatment regimen. The use of single-pill combinations has been shown to markedly improve patient adherence [43-45]. Improved adherence is associated with greater BP control $[46,47]$ and reduced cardiovascular events [48]. People with hypertension often suffer other comorbid conditions that may necessitate taking multiple medications. Healthcare providers and patients may therefore be less willing to accept additional pills to treat one condition or to increase the dose of a single agent because of concerns about increased side-effects. Single-pill triple-combination therapy can help overcome this clinical inertia by reducing dosing complexity at the same time as improving efficacy and tolerability [49-51].

\section{Limitations}

Our study is subject to potential limitations. While randomized, the study was not conducted double blind according to the purpose of the study and its exploratory nature which compared fixed versus free combination regimens on a limited number of patients, consequently, no sample size calculation was performed and no formal power calculation were made. The type I error for statistical analyses was set at $\alpha=5 \%$ using a two-tailed test and 
no interpretation of statistical tests was provided. Due to the design of such randomized clinical trials, the enhanced compliance/adherence to treatment that would be expected with fixed combination cannot be determined; only a clinical study conducted in daily medical practice could address this issue. However, control of hypertension was demonstrated from the week 4 visit in $88 \%$ of patients on the single-pill triple-drug combination compared with $79 \%$ on dual therapy + amlodipine. Finally, only the lowest dose of this fixed combination was evaluated (Per/Ind/Aml 5/1.25/5 mg). Two higher, single-pill, triple-therapy dosages (10/ $2.5 / 5$ and $10 / 2.5 / 10 \mathrm{mg}$ ) are also available on the market.

\section{CONCLUSIONS}

In patients with uncontrolled essential hypertension, single-pill triple therapy with perindopril $5 \mathrm{mg} /$ indapamide $1.25 \mathrm{mg} /$ amlodipine $5 \mathrm{mg}$ was as effective as the same dose of a dual-pill combination of perindopril/indapamide plus separate amlodipine with earlier achievement of BP control.

Both dosing regimens produced clinically significant reductions in SBP and DBP at 1 month which were maintained until the end of the study. The majority of patients in both treatment groups responded early to the antihypertensive therapy and achieved BP control. Both treatment regimens were well tolerated.

Until now, the only choice for physicians wanting to initiate single-pill triple combinations was an ARB-based therapy $[1,2,6]$. Patients already treated with an ACE inhibitor-based dual therapy who required three drugs in a single-pill were obliged to switch to other therapeutic classes. Perindopril/indapamide/ amlodipine is currently the only triple-drug therapy incorporating an ACE inhibitor in a single pill, providing an evidence-based drug combination supported by international guidelines and recommendations for the treatment of hypertension.

\section{ACKNOWLEDGEMENTS}

This study and article processing charges were supported by Les Laboratoires Servier. All authors had full access to all of the data in this study and take complete responsibility for the integrity of the data and accuracy of the data analysis. All named authors meet the International Committee of Medical Journal Editors (ICMJE) criteria for authorship for this manuscript, take responsibility for the integrity of the work as a whole, and have given final approval for the version to be published.

Editorial assistance was provided by Jenny Grice and funded by Les Laboratoires Servier.

Sergey V. Nedogoda and Vesna J. Stojanov were supported for writing the study by Les Laboratoires Servier.

Disclosures. Sergey V. Nedogoda has received consulting fees or honorarium from Novartis, Sanofi-Aventis, Servier, AstraZeneca, Boehringer Ingelheim and Bayer. He has also received payment for lectures from Novartis, MSD, Sanofi-Aventis, Pfizer, Servier, Boehringer Ingelheim, Bayer, AstraZeneca, Abbott, and Takeda.

Vesna J. Stojanov has no conflicts of interest to declare.

Compliance with Ethics Guidelines. All procedures followed were in accordance with the ethical standards of the responsible committee on human experimentation (institutional and national) and with the Helsinki Declaration of 1964, as revised in 2013. Informed consent was obtained from all patients for being included in the study.

Data Availability. The datasets analyzed during the current study are available from the corresponding author on reasonable request.

Open Access. This article is distributed under the terms of the Creative Commons Attribution-NonCommercial 4.0 International License (http://creativecommons.org/licenses/ 
by-nc/4.0/), which permits any noncommercial use, distribution, and reproduction in any medium, provided you give appropriate credit to the original author(s) and the source, provide a link to the Creative Commons license, and indicate if changes were made.

\section{REFERENCES}

1. Mancia G, Fagard R, Narkiewicz K, Redón J, Zanchetti A, Böhm M, et al. Guidelines for the Management of Arterial Hypertension. The task force for the management of arterial hypertension of the European Society of Hypertension (ESH) and of the European Society of Cardiology (ESC). J Hypertens. 2013;31:1281-357.

2. James PA, Oparil S, Carter BL, Cushman WC, Dennison-Himmelfarb C, Handler J, et al. 2014 evidence-based guideline for the management of high blood pressure in adults: report from the panel members appointed to the Eighth Joint National Committee (JNC 8). JAMA. 2014;311:507-20.

3. Weber MA, Schiffrin EL, White WB, Mann S, Lindholm LH, Kenerson JG, et al. Clinical practice guidelines for the management of hypertension in the community a statement by the American Society of Hypertension and the International Society of Hypertension. J Hypertens. 2014;32:3-15.

4. National Institute for Health and Care Excellence. Hypertension in adults: diagnosis and management. Clinical Guidelines CG127. 2011.

5. Benoit G, Feber J, Harris KD, Poirier L, Padwal RS, For the Canadian Hypertension Education Program. The 2015 Canadian Hypertension Education Program recommendations for blood pressure measurement, diagnosis, assessment of risk, prevention, and treatment of hypertension. Can J Cardiol. 2015;31:549-68.

6. Tykarski A, Narkiewicz K, Gaciong Z, Polish Society of Hypertension, et al. 2015 guidelines for the management of hypertension. Recommendations of the Polish Society of Hypertension. Arterial Hypertens. 2015;19:53-83.

7. Mancia G, De Backer G, Dominiczak A, The task force for the management of arterial hypertension of the European Society of Hypertension, The task force for the management of arterial hypertension of the European Society of Cardiology, et al. 2007 Guidelines for the management of arterial hypertension: the Task Force for the Management of Arterial Hypertension of the European Society of
Hypertension (ESH) and of the European Society of Cardiology (ESC). Eur Heart J. 2007;28:1462-1536.

8. Wald DS, Law M, Morris JK, Bestwick JP, Wald NJ. Combination therapy vs. monotherapy in reducing blood pressure: meta-analysis on 11,000 participants from 42 trials. Am J Med. 2009;122:290-300.

9. Leung AA, Nerenberg K, Daskalopoulou SS, CHEP Guidelines Task Force, et al. Hypertension Canada's 2016 Canadian Hypertension Education Program guidelines for blood pressure measurement, diagnosis, assessment of risk, prevention, and treatment of hypertension. Can J Cardiol. 2016;32:569-88.

10. Gradman AH. Rationale for triple-combination therapy for management of high blood pressure. J Clin Hypertens (Greenwich). 2010;12:869-78.

11. Elijovich F, Laffer C. A role for single-pill triple therapy in hypertension. Ther Adv Cardiovasc Dis. 2009;3:231-40.

12. Black HR. Triple fixed-dose combination therapy. Back to the past. Hypertension. 2009;54:19-22.

13. Neutel JM, Smith DH. Hypertension management: rationale for triple therapy based on mechanisms of action. Cardiovasc Ther. 2013;31:251-8.

14. Makani $\mathrm{H}$, Bangalore $\mathrm{S}$, Romero J, Wever-Pinzon $\mathrm{O}$, Messerli FH. Effect of renin-angiotensin system blockade on calcium channel blocker-associated peripheral edema. Am J Med. 2011;124:128-35.

15. Chung N, Baek S, Chen MF, et al. Expert recommendations on the challenges of hypertension in Asia. Int J Clin Pract. 2008;62:1306-12.

16. Remme WJ, McMurray JJ, Hobbs FD, et al. Awareness and perception of heart failure among European cardiologists, internists, geriatricians, and primary care physicians. Eur Heart J. 2008;29:1739-52.

17. Liu L. 2010 Chinese guidelines for the management of hypertension. Chin J Cardiol. 2011;39:579-616.

18. Sociedade Brasileira de Cardiologia (SBC). 7a Diretriz Brasileira de Hipertensão Arterial. Arq Bras Cardiol. 2016;107(3 Suppl.3):1-83.

19. Piepoli MF, Hoes AW, Agewall S, et al. 2016 European Guidelines on cardiovascular disease prevention in clinical practice. The Sixth Joint Task Force of the European Society of Cardiology and Other Societies on Cardiovascular Disease Prevention in Clinical Practice (constituted by representatives of 10 societies and by invited experts). Eur Heart J. 2016; . doi:10.1093/eurheartj/ehw106. 
20. Russian Society on Hypertension. All-Russian Scientific Society of Cardiologists (VNOK). Diagnosis and treatment of arterial hypertension. Systemnye Hypertensii. 2010;3:5-26.

21. Fogari R, Zoppi A, Tettamanti F, Malamani GD, Tinelli C, Salvetti A. Effects of nifedipine and indomethacin on cough induced by angiotensin-converting enzyme inhibitors: a double-blind, randomized, cross-over study. J Cardiovasc Pharmacol. 1992;19:670-3.

22. Tóth K, Investigators PIANIST. Antihypertensive efficacy of triple combination perindopril/indapamide plus amlodipine in high-risk hypertensives: results of the PIANIST study (Perindopril-Indapamide plus AmlodipiNe in high rISk hyperTensive patients). Am J Cardiovasc Drugs. 2014;14:137-45.

23. PROGRESS Collaborative Group. Randomised trial of a perindopril-based blood-pressure-lowering regimen among 6,105 individuals with previous stroke or transient ischaemic attack. Lancet. 2001;358:1033-41.

24. ADVANCE Collaborative Group. Effect of a fixed combination of perindopril and indapamide on macrovascular and microvascular outcomes in patients with type 2 diabetes mellitus (the ADVANCE trial): a randomized controlled trial. Lancet. 2007;370:829-40.

25. Beckett $\mathrm{N}$, Peters $\mathrm{R}$, Fletcher AE, For the HYVET Study Group, et al. Treatment of hypertension in patients 80 years of age or older. N Engl J Med. 2008;358:1887-98.

26. Dahlof B, Sever P, Poulter N, For the ASCOT investigators, et al. Prevention of cardiovascular events with an antihypertensive regimen of amlodipine adding perindopril as required versus atenolol adding bendroflumethiazide as required, in the Anglo-Scandinavian Cardiac Outcomes Trial-Blood Pressure Lowering Arm (ASCOT-BPLA): a multicentre randomised controlled trial. Lancet. 2005;366:895-90.

27. Julius S, Kjeldsen SE, Weber M, VALUE trial group, et al. Outcomes in hypertensive patients at high cardiovascular risk treated with regimens based on valsartan or amlodipine: the VALUE randomised trial. Lancet. 2004;363:2022-31.

28. The ALLHAT Officers and Coordinators for the ALLHAT Collaborative Research Group. Major outcomes in high-risk hypertensive patients randomized to angiotensin-converting enzyme inhibitor or calcium channel blocker vs diuretic: the Antihypertensive and Lipid-Lowering treatment to prevent Heart Attack Trial (ALLHAT). JAMA. 2002;288:2981-97.
29. Jamerson K, Bakris GL, Dahlöf B, ACCOMPLISH Investigators, et al. Exceptional early blood pressure control rates: the ACCOMPLISH trial. Blood Press. 2007;16:80-6.

30. Chalmers J, Arima H, Woodward M, et al. Effects of combination of perindopril, indapamide, and calcium channel blockers in patients with type 2 diabetes mellitus: results from the Action In Diabetes And Vascular Disease: Preterax and Diamicron Controlled Evaluation (ADVANCE) trial. Hypertension. 2014;63:259-64.

31. de la Sierra A. Mitigation of calcium channel blocker-related oedema in hypertension by antagonists of the renin-angiotensin system. J Hum Hypertens. 2009;23:503-11.

32. Oparil S, Melino M, Lee J, Fernandez V, Heyrman R. Triple therapy with olmesartan medoxomil, amlodipine besylate, and hydrochlorothiazide in adult patients with hypertension: the TRINITY multicenter, randomized, double-blind, 12-week, parallel-group study. Clin Ther. 2010;32:1252-69.

33. Calhoun DA, Crikelair NA, Yen J, Glazer RD. Amlodipine/valsartan/hydrochlorothiazide triple combination therapy in moderate/severe hypertension: secondary analyses evaluating efficacy and safety. Adv Ther. 2009;26:1012-23.

34. Chen P, Chaugai S, Zhao F, Wang DW. Cardioprotective effect of thiazide-like diuretics: a meta-analysis. Am J Hypertens. 2015;28:1453-63.

35. Kaplan N. Treatment of hypertension: drug therapy-diuretics. In: Kaplan NM, editor. Clinical hypertension, 8th ed. Philadelphia, USA: Lippincott Williams and Wilkins; 2002. p. 241-252.

36. Messerli FH, Makani H, Benjo A, Romero J, Alviar C, Bangalore S. Antihypertensive efficacy of hydrochlorothiazide as evaluated by ambulatory blood pressure monitoring. A meta-analysis of randomized trials. J Am Coll Cardiol. 2011;57:590-600.

37. NICE-Clinical Guidelines Hypertension: the clinical management of primary hypertension in adults-NCGC (National Clinical Guideline Centre) 127. 2011.

38. Roush GC, Ernst ME, Kostis JB, Tandon S, Sica DA. Head-to-head comparisons of hydrochlorothiazide with indapamide and chlorthalidone antihypertensive and metabolic effects. Hypertension. 2015;65:1041-6.

39. Ferrari R, Rosano GM. Not just numbers, but years of science: putting the ACE inhibitor-ARB meta-analyses into context. Int $\mathrm{J}$ Cardiol. 2013;166:286-8. 
40. Van Vark LC, Bertrand M, Akkerhuis KM, et al. Angiotensin-converting enzyme inhibitors reduce mortality in hypertension: a meta-analysis of randomized clinical trials of renin-angiotensin-aldosterone-system inhibitors involving 158,998 patients. Eur Heart J. 2012;33:2088-97.

41. Savarese G, Costanzo P, Cleland JG, et al. A meta-analysis reporting effects of angiotensin-converting enzyme inhibitors and angiotensin receptor blockers in patients without heart failure. J Am Coll Cardiol. 2013;61:131-42.

42. Strauss MH, Hallb AS. The divergent cardiovascular effects of angiotensin converting enzyme inhibitors and angiotensin receptor blockers on myocardial infarction and death. Prog Cardiovasc Dis. 2016;58:473-82.

43. Gupta AK, Arshad S, Poulter NR. Compliance, safety, and effectiveness of fixed-dose combinations of antihypertensive agents: a meta-analysis. Hypertension. 2010;55:399-407.

44. Dragomir A, Cote R, Roy L, et al. Impact of adherence to antihypertensive agents on clinical outcomes and hospitalization costs. Med Care. 2010;48:418-25.

45. Bangalore S, Kamalakkannan G, Parkar S, Messerli FH. Fixed-dose combinations improve medication compliance: a meta-analysis. Am J Med. 2007;120:713-9.
46. Bramley TJ, Gerbino PP, Nightengale BS, Frech-Tamas F. Relationship of blood pressure control to adherence with antihypertensive monotherapy in 13 managed care organizations. J Manag Care Pharm. 2006;12:239-45.

47. Yiannakopoulou EC, Papadopulos JS, Cokkinos DV, et al. Adherence to antihypertensive treatment: a critical factor for blood pressure control. Eur J Cardiovasc Prev Rehabil. 2005;12:243-9.

48. Mazzaglia G, Ambrosioni E, Alacqua M, et al. Adherence to antihypertensive medications and cardiovascular morbidity among newly diagnosed hypertensive patients. Circulation. 2009;120:1598-605.

49. Heisler M, Hogan MM, Hofer TP, Schmittdiel JA, Pladevall M, Kerr EA. When more is not better: treatment intensification among hypertensive patients with poor medication adherence. Circulation. 2008;117:2884-92.

50. Okonofua EC, Simpson KN, Jesri A, Rehman SU, Durkalski VL, Egan BM. Therapeutic inertia is an impediment to achieving the Healthy People 2010 blood pressure control goals. Hypertension. 2006;47:345-51.

51. Basile J, Neutel J. Overcoming clinical inertia to achieve blood pressure goals: the role of fixed-dose combination therapy. Ther Adv Cardiovasc Dis. 2010;4:119-27. 